\title{
AVALIAÇÃO DA QUALIDADE DE VIDA DE ADOLESCENTES EM SITUAÇÃO DE VULNERABILIDADE SOCIAL
}

\section{Quality of life assessment among socially vulnerable adolescents}

\author{
Evaluación de la calidad de vida de adolescentes en situación \\ de vulnerabilidad social
}

\section{RESUMO}

Objetivo: Avaliar a qualidade de vida de adolescentes em situação de vulnerabilidade social. Métodos: Estudo exploratório quantitativo, realizado de novembro de 2014 a fevereiro de 2015, em Recife, Pernambuco, com 86 adolescentes de ambos os sexos. Utilizou-se o questionário Kidscreen-52, que avalia e mensura a saúde subjetiva relacionada à qualidade de vida (QV), através de dez dimensões: "Saúde e atividade física"; "Sentimentos"; "Estado de humor global"; "Autopercepção"; "Autonomia/Tempo livre"; "Família e ambiente familiar"; "Questões econômicas"; “Amigos"; "Ambiente escolar e aprendizagem"; e Provocação/Bullying - todas analisadas com teste " $t$ ", sendo $p<0,05$. Resultados: As dimensões "Sentimentos" ( $\dot{\mathrm{x}}=86,01)$ e "Provocação/Bullying" $(\dot{\mathrm{x}}=85,59)$ apresentaram melhor percepção; já os domínios "Aspectos financeiros" ( $\dot{\mathrm{x}}=66,43)$ e "Autopercepção" $(\dot{x}=72,62)$ apresentaram pior percepção. Quando comparado os domínios por sexo, houve diferença significativa nas dimensões "Saúde e atividade física" ( $p=0,0051)$, "Sentimentos" $(p=0,0342)$, "Estado de humor global" $(p=0,0226)$, “Autonomia e tempo livre" $(p=0,0287)$, "Família e ambiente familiar" ( $p=0,0077)$ e "Amigos e apoio social" ( $p=0,0058)$, apontando melhor percepção para o sexo masculino. Conclusão: Os adolescentes pesquisados possuem boa percepção da qualidade de vida $(\mathrm{QV})$, porém, o sexo masculino apresentou melhor percepção em todos os domínios, e isso interfere diretamente na sua QV.

Descritores: Desenvolvimento do Adolescente; Qualidade de Vida; Vulnerabilidade Social.

\section{ABSTRACT}

Objective: To assess the quality of life of socially vulnerable adolescents. Methods: Quantitative exploratory study conducted from November 2014 to February 2015 in Recife, Pernambuco, with 86 adolescents of both genders. The Kidscreen-52 questionnaire was used to assess and measure the subjective health-related quality of life (QoL) through ten dimensions: "Physical Well-being"; "Psychological Well-being"; "Moods and Emotions"; "Self-perception"; "Autonomy"; "Parent Relations and Home Life"; "Financial Resources"; "Social Support and Peers"; "School Environment"; and "Social Acceptance /Bullying" - all of them were analyzed using $t$ test, with $p<0.05$. Results: The dimensions "Psychological Well-being" $(x=86.01)$ and Social Acceptance/Bullying $(x=85.59)$ presented the best rates; on the other hand, "Financial Resources" ( $x=66.43)$ and "Self-perception" $(x=72.62)$ presented the worst rates. With regard to the domains by sex, significant difference was found in the dimensions "Physical Well-being" $(p=0.0051)$, "Psychological Wellbeing" ( $p=0.0342)$, "Moods and Emotions" ( $p=0.0226)$, "Autonomy" ( $p=0.0287)$, "Parent Relations and Home Life" ( $p=0.0077)$ and "Social Support and Peers" $(p=0.0058)$, indicating a better perception by male participants. Conclusion: The adolescents assessed have a good perception of QoL; however, the boys showed better perception in all domains, and this directly affects their QoL.

NCT: 28559614.8.0000.5208

Descriptors: Adolescent Development; Quality of Life; Social Vulnerability.

\section{Artigo Original}

1) Universidade Federal de Pernambuco UFPE - Recife - (PE) - Brasil

2) Universidade Rural de Pernambuco UFRPE - Recife - (PE) - Brasil

3) Centro de Atenção Psicossocial Infantojuvenil - CAPSi - Recife (PE) - Brasil
Recebido em: 11/09/2015 Revisado em: 10/11/2015 Aceito em: 01/12/2015 


\section{RESUMEN}

Objetivo: Evaluar la calidad de vida de adolescentes en situación de vulnerabilidad social. Métodos: Estudio exploratorio cuantitativo realizado entre noviembre de 2014 y febrero de 2015 en Recife, Pernambuco, con 86 adolescentes de ambos los sexos. Se utilizó el cuestionario Kidscreen-52 que evalúa y mide la salud subjetiva relacionada a la calidad de vida (CV) a través de diez dimensiones: "Salud y actividad fisica"; "Sentimientos"; "Estado de humor general"; "Auto percepción”; "Autonomíal Tiempo libre”; "Familia y ambiente familiar"; "Cuestiones económicas"; "Amigos"; "Ambiente escolar y aprendizaje"; $y$ "Provocación/Bullying - todas analizadas con el teste " $t$ " $y$ $p<0,05$. Resultados: Las dimensiones "Sentimientos" $(\dot{x}=86,01)$ $y$ "Provocación/Bullying" $(\dot{x}=85,59)$ presentaron mejor percepción; los dominios "Aspectos financieros" ( $\dot{x}=66,43) y$ "Auto percepción” $(\dot{x}=72,62)$ presentaron peor percepción. Hubo diferencia significativa para las dimensiones "Salud y actividad física" $(p=0,0051)$, "Sentimientos" ( $p=0,0342)$, "Estado de humor general” ( $p=0,0226)$, “Autonomía y tiempo libre” ( $p=0,0287)$, "Familia y ambiente familiar" ( $p=0,0077) y$ "Amigos y apoyo social” ( $p=0,0058)$ al comparar los dominios por sexo con mejor percepción en el sexo masculino. Conclusión: Los adolescentes investigados tienen buena percepción de la calidad de vida (CV), sin embargo, el sexo masculino presentó mejor percepción para todos los dominios lo que interfiere directamente en su $\mathrm{CV}$.

Descriptores: Desarrollo del Adolescente; Calidad de Vida; Vulnerabilidad Social.

\section{INTRODUÇÃO}

A Organização Mundial de Saúde considera adolescência entre 10 e 19 anos de idade ${ }^{(1)}$. No Brasil, o Estatuto da Criança e do Adolescente (ECA) - Lei 8.069, de 1990 - define a adolescência como o período compreendido entre 12 e 18 anos de idade, marcado por mudanças, desenvolvimentos e desafios que podem interferir nos aspectos físicos, psicológicos e sociais da vida dos sujeitos ${ }^{(2)}$.

A adolescência se configura como um período de experimentação de valores, papéis sociais e identidade, além de ser caracterizada como uma fase evolutiva, única e exclusiva, em que acontecem intensas e profundas transformações físicas, mentais e sociais, sendo uma importante etapa para a formação da personalidade ${ }^{(3)}$.

Considerando todas as crianças e adolescentes como sujeitos de direitos nas diversas condições sociais e individuais, o ECA preconiza o direito à vida, à saúde, à alimentação, à educação, ao lazer, à profissionalização, à cultura, à dignidade, ao respeito, à liberdade e à convivência familiar e comunitária, além de tentar colocá-los a salvo de toda forma de negligência, discriminação, exploração, violência, crueldade e opressão. Além disso, o ECA destaca a inviolabilidade da integridade física, psíquica e moral, abrangendo a identidade, a autonomia, os valores, as ideias, o direito de opinião e expressão, de buscar refúgio, auxílio e orientação ${ }^{(4)}$.

Devido à sua condição de pessoa em desenvolvimento e às mudanças que ocorrem nessa fase, o adolescente traz em si uma condição intrínseca de vulnerabilidade, necessitando, assim, de proteção e cuidados físicos, psíquicos e morais ${ }^{(5,6)}$. Entre as diferentes vulnerabilidades às quais os adolescentes podem estar susceptíveis, a vulnerabilidade social encontra um lugar de destaque nas discussões do meio acadêmico e social. A vulnerabilidade social é um conceito multidimensional que caracteriza a existência de indivíduos, grupos ou lugares em situação de fragilidade, seja por fatores biológicos, epidemiológicos, sociais e/ou culturais. Esses fatores tornam os indivíduos expostos a riscos e a níveis significativos de desagregação social que acabam por influenciar no seu modo de viver e de adoecer, e, consequentemente, na sua qualidade de vida $(\mathrm{QV})^{(5-7)}$.

A definição de QV está relacionada com a percepção que o indivíduo possui da sua posição na vida, dos seus objetivos, expectativas, contexto cultural e padrões em que vive. É um conceito amplo, afetado de forma complexa pela saúde física, pelo estado psicológico, nível de dependência, relações sociais, crenças pessoais e ambiente em que vive $^{(8,9)}$.

O grupo europeu Kidscreen-52 defende que a QV é multidimensional e é vista como uma construção psicológica que descreve aspectos físicos, mentais, psicológicos, funcionais e sociais do bem-estar. Nessa perspectiva, adotada no presente trabalho, é importante analisar os vários fatores envolvidos nessa fase da vida, a adolescência, uma vez que eles podem funcionar como fatores protetores ou fatores de risco da QV dessa população ${ }^{(10)}$.

Frequentemente, a literatura discute a QV relacionando-a a alguma patologia, sem considerar o bem-estar de pessoas saudáveis, porém, esse cenário está mudando e o tema começa a abranger os aspectos físicos, psicológicos e sociais, as áreas funcionais da vida e o impacto da saúde e da doença sobre esses setores, tratando das condições gerais de uma população, assim como de um grupo ou indivíduo específico ${ }^{(11)}$.

Especificamente no que se refere à QV na adolescência, estudo realizado com escolares de uma comunidade vulnerável do sul do Brasil mostrou a importância do apoio familiar, pois se observou que a QV dos escolares sofre influência da família no desejo e nas escolhas de estudar e trabalhar, pois ela é referência para atitudes, comportamentos e valores. Além disso, as condições sociais, como a renda familiar e a escolaridade dos familiares, são fatores que interferem na $\mathrm{QV}^{(12)}$. 
Outras pesquisas realizadas com adolescentes em risco social demonstraram que a percepção acerca da QV envolve o suprimento das necessidades humanas básicas e atributos valorizados pela sociedade contemporânea ${ }^{(13,14)}$. Aspectos objetivos, como segurança, nutrição adequada, higiene, esportes e condição de vida satisfatória, além de construtos subjetivos, como afetividade na amizade, nos relacionamentos amorosos e na família, são considerados essenciais pelos adolescentes para ter uma boa QV. Além disso, ter QV, para esses jovens, envolve sua posição enquanto sujeitos de direitos no contexto familiar e social; e quando comparados a adolescentes que não apresentam características de risco social, eles apresentam uma melhor percepção nas dimensões de autonomia e relação com os pais $^{(13,14)}$

Considerando que a adolescência é afetada por vários fatores e que isso interfere diretamente na $\mathrm{QV}$, é importante a realização de estudos com adolescentes em diferentes contextos de vida, podendo contribuir para uma compreensão mais aprofundada da situação em que se encontram e uma análise dos fatores que possuem relação direta com a QV, a fim de fortalecer ações dedicadas à promoção dos direitos das crianças e dos adolescentes em situação de vulnerabilidade. Desse modo, o objetivo deste estudo é avaliar a qualidade de vida de adolescentes em situação de vulnerabilidade social.

\section{MÉTODOS}

Estudo exploratório, com abordagem quantitativa, realizado no período de novembro de 2014 a fevereiro de 2015 com adolescentes residentes na cidade de Recife, Pernambuco.

Trata-se de uma das etapas do projeto de pesquisa intitulado "Qualidade de vida de adolescentes participantes de um programa social de esporte educacional", desenvolvido com participantes de um programa social de esporte educacional, o qual oferece múltiplas vivências esportivas no contraturno escolar e tem como objetivo promover o desenvolvimento integral de crianças, adolescentes e jovens como fator de formação da cidadania e melhoria da QV, prioritariamente daqueles que se encontram em áreas de vulnerabilidade social e regularmente matriculados na rede pública de ensino ${ }^{(15,16)}$

Coletaram-se os dados em quatro núcleos, sendo convidados a participar do estudo os adolescentes alfabetizados, inscritos no programa, com idade entre 12 e 18 anos incompletos, de ambos os sexos, sem deficiência físicas e com autorização de seus responsáveis legais. No período da coleta de dados, os núcleos contavam com 142 adolescentes, dos quais 86 participaram do estudo. Destacase que não foi possível a inclusão de um número maior de participantes devido à dificuldade de se conseguir o Termo de Consentimento Livre e Esclarecido (TCLE) assinado pelos pais. Houve recusa de dois adolescentes.

Utilizou-se como instrumento o questionário Kidscreen-52. Inicialmente, obteve-se a assinatura do TCLE dos pais e adolescentes; em seguida, o questionário foi respondido pelos adolescentes, com orientação de uma equipe de pesquisa devidamente treinada, sendo impresso e aplicado nas salas das escolas onde o programa era operacionalizado.

O Kidscreen-52 é um instrumento transcultural europeu, traduzido e adaptado para uso no Brasil( ${ }^{(10)}$, desenvolvido nos anos 2001-2004, que mede a saúde geral associada à QV de crianças e adolescentes. Além disso, descreve as variáveis demográficas (sexo e idade), o estado de saúde física, mental e social, a relação dos adolescentes com os pais e o suporte social ${ }^{(10,17)}$.

É um questionário de autopreenchimento, que contém 52 questões objetivas, com tempo médio de aplicação entre 10 e 15 minutos, sendo constituído por dez dimensões: "Saúde e atividade física"; "Sentimentos"; "Estado de humor global"; "Autopercepção"; "Autonomia/Tempo livre"; "Família e ambiente familiar"; "Questões econômicas"; "Amigos"; "Ambiente escolar e aprendizagem"; e "Provocação/Bullying"(10). Cada uma das dimensões continha de 3 a 6 questões, que poderiam ser respondidas a partir de uma escala tipo Likert de cinco pontos, na qual o adolescente assinala: 1=nada/nunca, $2=$ pouco/ raramente, $3=$ moderadamente/algumas vezes, $4=$ muito/ frequentemente, e $5=$ totalmente/sempre. As pontuações de cada item variam de 1 a 5 , e o escore total varia entre 52 e 260 pontos - as pontuações mais altas indicam melhor QV relacionada à saúde ${ }^{(10)}$.

Os dados provenientes dos questionários preenchidos foram digitados em planilha Excel 2010, validados por meio do sistema de dupla digitação e analisados com auxílio do Programa Biostatic. Assim como em outro estudo ${ }^{(8)}$, analisaram-se os dados da seguinte maneira: as questões 1.1 , todas as questões da dimensão 3 , as questões $4.4,4.5$ e todas as questões da dimensão 10 tiveram sua pontuação invertida $(1=5,2=4,3=3,4=2$ e $5=1)$, pois a escala é inversa. Essa inversão é realizada para promover a homogeneização dos resultados, de modo que, para todos os itens, um valor maior reflita uma QV mais elevada ${ }^{(10)}$. Em seguida, criou-se uma variável com a soma das pontuações em cada questão para cada participante, a qual se considerou como sendo o Escore Total (ET) do Kidscreen-52. Tendo em vista que a maior soma de todas as pontuações é 260 , o ET foi transformado para uma pontuação proporcional, sendo 260 igual a $100(\mathrm{ET} \%=\mathrm{ET} \times 100 / 260)$. Também foram criadas outras 10 variáveis, de acordo com o número de dimensões do Kidscreen-52, que são a soma das pontuações em cada questão para cada participante dentro de cada dimensão. 
Converteram-se as dez variáveis proporcionalmente, conforme descrito anteriormente.

Para se testar a normalidade das variáveis, utilizouse o teste de Kolmogorov Smirnov. A comparação entre as médias dos sexos para o escore total e para cada dimensão ocorreu por meio do teste " $t$ ", sendo adotado o nível de significância de $\mathrm{p}<0,05$.

Respeitaram-se os aspectos éticos durante o desenvolvimento da presente pesquisa, conforme preconizado pela Resolução 466/12 do CONEP, sendo o projeto aprovado pelo Comitê de Ética em Pesquisa com Seres Humanos da UFPE (parecer $n^{\circ}$. 651.839). Os instrumentos da pesquisa foram codificados a fim de se garantir o anonimato dos participantes da pesquisa.

\section{RESULTADOS}

Participaram do estudo 86 adolescentes do sexo feminino e do masculino, com idades compreendidas entre os 12 e os 18 anos incompletos, com média de idade de 14,3 anos. Somente dois adolescentes apontaram a convivência com doenças que exigem cuidados de saúde esporádicos (alergia e neuropatia) (Tabela I).

No que se refere à percepção da $\mathrm{QV}$ e dos fatores a ela relacionados, na Tabela II são apresentadas pontuações máximas e mínimas, a média geral dos adolescentes em cada domínio e no instrumento como um todo. Observa-se que, no escore total, os adolescentes demonstraram uma boa percepção da QV, com média na pontuação de $66 \%$, o que corresponde a 172 pontos no total de 260 do instrumento.

A análise de cada domínio permitiu a identificação dos aspectos com maior e menor impacto na QV dos adolescentes. Nesse sentido, identificou-se que a dimensão "Sentimentos" ( $\dot{\mathrm{x}}=86,01)$ e a dimensão "Provocação/ Bullying" ( $\dot{\mathrm{x}}=85,59)$ apresentaram melhor percepção; já os domínios "Aspectos financeiros" $(\dot{\mathrm{x}}=66,43)$ e "Autopercepção" ( $\dot{\mathrm{x}}=72,62)$ apresentaram as médias mais baixas.

A análise comparativa dos dados considerando o sexo está explicitada na Tabela III. Com relação ao escore geral

Tabela I - Caracterização geral dos participantes do estudo. Recife, PE, 2014-2015.

\begin{tabular}{lcc}
\hline Variáveis & $\mathbf{n}$ & $\mathbf{\%}$ \\
\hline Sexo & & \\
Feminino & 38 & 44,19 \\
Masculino & 48 & 55,81 \\
Idade (anos) & & \\
12 & 15 & 17,44 \\
13 & 19 & 22,09 \\
14 & 19 & 22,09 \\
15 & 7 & 8,14 \\
16 & 11 & 12,79 \\
17 & 14 & 16,28 \\
18 & 1 & 1,16 \\
Presença de doença crônica & & 97,67 \\
Não & 84 & 2,33 \\
Sim & 2 & \\
\hline
\end{tabular}

Tabela II - Distribuição das pontuações mínimas e máximas, médias e desvios-padrão nas dimensões do Kidscreen-52. Recife, PE, 2014-2015.

\begin{tabular}{lcccc}
\hline Dimensão & Mínima & Máxima & Média & Desvio Padrão \\
\hline Saúde e atividade física - D1 & 24,0 & 100,0 & 77,86 & 18,83 \\
Sentimentos - D2 & 26,67 & 100,0 & 86,01 & 17,25 \\
Estado emocional - D3 & 28,57 & 100,0 & 74,50 & 18,20 \\
Autopercepção - D4 & 32,0 & 100,0 & 72,62 & 17,69 \\
Autonomia e tempo livre - D5 & 20,0 & 100,0 & 80,65 & 19,54 \\
Família e ambiente familiar - D6 & 23,33 & 100,0 & 80,65 & 20,03 \\
Aspectos financeiros - D7 & 20,0 & 100,0 & 66,43 & 20,67 \\
Amigos e apoio social - D8 & 36,66 & 100,0 & 80,99 & 17,12 \\
AmbienteeEscolar - D9 & 43,33 & 100,0 & 79,10 & 16,45 \\
Provocação/Bullying - D10 & 33,33 & 100,0 & 85,59 & 19,83 \\
Escore total & 28,79 & 100,0 & 78,44 & 18,56 \\
\hline
\end{tabular}


obtido encontrou-se diferença estatisticamente significativa, na qual o sexo masculino apresentou melhor percepção da QV em contraposição ao feminino, assim como nas dimensões "Saúde e atividade física" ( $p=0,0051)$, "Sentimentos" $(p=0,0342)$, "Estado emocional" $(p=0,0226)$, "Autonomia e tempo livre" ( $p=0,0287)$, "Família e ambiente familiar" $(p=0,0077)$ e "Amigos e apoio social" $(p=0,0058)$. As diferenças em todas as dimensões apontam uma melhor QV para o sexo masculino.
Considerando que, conforme expresso na Tabela III e explicitado anteriormente, os domínios "saúde e atividade física", "família/ambiente familiar" e "amigos e apoio social" apresentaram as diferenças estatisticamente mais significativas, nas Tabelas IV e V são apresentadas as respostas dadas pelos adolescentes a cada questão do instrumento nesses âmbitos. Estas duas últimas dimensões citadas foram agrupadas na Tabela V.

Tabela III - Análise entre as dimensões do Kidscreen-52 em relação ao sexo dos participantes. Recife, PE, $2014-2015$.

\begin{tabular}{|c|c|c|c|c|c|}
\hline Dimensões & Sexo & $\mathbf{n}$ & Média & Desvio Padrão & Valor de p \\
\hline \multirow[t]{2}{*}{ Saúde e atividade física - D1 } & Masculino & 48 & 82,25 & 15,08 & $0,0051 * *$ \\
\hline & Feminino & 38 & 72,31 & 16,90 & \\
\hline \multirow{2}{*}{ Sentimentos - D2 } & Masculino & 48 & 88,48 & 11,58 & $0,0342 *$ \\
\hline & Feminino & 38 & 82,26 & 15,10 & \\
\hline \multirow{2}{*}{ Estado emocional - D3 } & Masculino & 48 & 77,62 & 15,11 & $0,0226^{*}$ \\
\hline & Feminino & 38 & 69,68 & 16,42 & \\
\hline \multirow{2}{*}{ Autopercepção - D4 } & Masculino & 48 & 74,50 & 15,97 & 0,2147 \\
\hline & Feminino & 38 & 70,26 & 15,14 & \\
\hline \multirow{2}{*}{ Autonomia e tempo livre - D5 } & Masculino & 48 & 84,25 & 16,41 & $0,0287 *$ \\
\hline & Feminino & 38 & 76,10 & 17,41 & \\
\hline \multirow{2}{*}{ Família e ambiente familiar - D6 } & Masculino & 48 & 85,52 & 14,79 & $0,0077 * *$ \\
\hline & Feminino & 38 & 75,34 & 19,76 & \\
\hline \multirow{2}{*}{ Aspectos financeiros - D7 } & Masculino & 48 & 69,60 & 18,76 & 0,0614 \\
\hline & Feminino & 38 & 61,66 & 20,10 & \\
\hline \multirow{2}{*}{ Amigos e apoio social - D8 } & Masculino & 48 & 84,52 & 10,69 & $0,0058 * *$ \\
\hline & Feminino & 38 & 75,84 & 16,11 & \\
\hline \multirow{2}{*}{ Ambiente familiar - D9 } & Masculino & 48 & 79,37 & 14,0 & 0,6370 \\
\hline & Feminino & 38 & 78,00 & 12,51 & \\
\hline \multirow{2}{*}{ Provocação/Bullying - D10 } & Masculino & 48 & 87,52 & 16,92 & 0,1862 \\
\hline & Feminino & 38 & 82,55 & 17,30 & \\
\hline \multirow{2}{*}{ Total } & Masculino & 48 & 81,46 & 14,93 & $0,0007 * *$ \\
\hline & Feminino & 38 & 74,45 & 16,67 & \\
\hline
\end{tabular}

${ }^{*} \mathrm{p} \leq 0,05 * * \mathrm{p} \leq 0,01$

Tabela IV - Respostas por sexo das questões do domínio "Saúde e atividade física”. Recife, PE, 2014-2015.

\begin{tabular}{lccccccc}
\hline \multirow{2}{*}{ Variáveis } & \multirow{2}{*}{ Sexo } & \multicolumn{2}{c}{$\begin{array}{c}\text { Nunca/ } \\
\text { Quase nunca }\end{array}$} & \multicolumn{2}{c}{$\begin{array}{c}\text { Moderadamente/ } \\
\text { Boa }\end{array}$} & \multicolumn{2}{c}{$\begin{array}{c}\text { Quase sempre/ } \\
\text { Sempre }\end{array}$} \\
\cline { 3 - 8 } & & $\mathbf{n}$ & $\mathbf{\%}$ & $\mathbf{n}$ & $\mathbf{\%}$ & $\mathbf{n}$ & $\mathbf{\%}$ \\
\hline \multirow{2}{*}{ Você se sentiu bem e em forma? } & Feminino & 4 & 10,53 & 13 & 34,21 & 21 & 55,27 \\
& Masculino & 6 & 12,50 & 6 & 12,50 & 36 & 75,00 \\
Você foi ativo fisicamente (correun & Feminino & 12 & 31,58 & 5 & 13,16 & 21 & 55,26 \\
andou de bicicleta)? & Masculino & 9 & 18,75 & 4 & 8,33 & 35 & 72,92 \\
Você foi capaz de correr? & Feminino & 10 & 26,32 & 9 & 23,68 & 19 & 50,00 \\
Você se sentiu com muita energia/ & Masculino & 12 & 27,09 & 2 & 4,12 & 33 & 68,75 \\
disposição? & Feminino & 4 & 10,52 & 13 & 34,21 & 21 & 55,26 \\
& Masculino & 0 & 0,00 & 9 & 18,75 & 39 & 81,25 \\
\hline
\end{tabular}


Quanto ao domínio "família/ambiente familiar", destaca-se a seguinte pergunta: "Seus pais entendem você?". Somente 17 (46,37\%) adolescentes do sexo feminino responderam "quase sempre/sempre", em contraposição a $36(75 \%)$ do sexo masculino. Na pergunta "Você conversou com seus pais como você gostaria?", 20 (52,63\%) adolescentes do sexo feminino responderam "quase/sempre ou sempre", enquanto $39(81,25 \%)$ adolescentes do sexo masculino optaram por essa resposta (Tabela V).
No domínio "Amigos e apoio social", quando indagados se eles tinham tempo suficiente para ficar com os amigos, 21 (54,26\%) adolescentes do sexo feminino responderam "quase sempre/sempre", sendo esta a resposta de $39(81,25 \%)$ dos adolescentes do sexo masculino. Para $19(50 \%)$ adolescentes do sexo feminino, a questão "Você sentiu que pode confiar em seus amigos" foi respondida como "quase sempre/sempre", resposta semelhante encontrada em $30(62,50 \%)$ questionários preenchidos por adolescentes do sexo masculino.

Tabela V - Respostas por sexo das questões dos domínios "Família e ambiente familiar" e "Amigos e apoio social”. Recife, PE, 2014-2015.

\begin{tabular}{|c|c|c|c|c|c|c|c|}
\hline \multirow[t]{2}{*}{ Variáveis } & \multirow[t]{2}{*}{ Sexo } & \multicolumn{2}{|c|}{$\begin{array}{c}\text { Nunca/ } \\
\text { Quase nunca }\end{array}$} & \multicolumn{2}{|c|}{$\begin{array}{l}\text { Algumas } \\
\text { vezes }\end{array}$} & \multicolumn{2}{|c|}{$\begin{array}{l}\text { Quase sempre/ } \\
\text { Sempre }\end{array}$} \\
\hline & & $\mathbf{n}$ & $\%$ & $\mathbf{n}$ & $\%$ & $\mathrm{n}$ & $\%$ \\
\hline \multirow[t]{2}{*}{ Seus pais entendem você? } & Feminino & 14 & 36,85 & 6 & 15,79 & 17 & 46,37 \\
\hline & Masculino & 3 & 6,25 & 9 & 18,75 & 36 & 75,00 \\
\hline \multirow{2}{*}{ Você se sentiu amado(a) pelos seus pais? } & Feminino & 3 & 7,89 & 4 & 10,53 & 31 & 81,58 \\
\hline & Masculino & 5 & 10,42 & 6 & 12,50 & 37 & 77,08 \\
\hline \multirow[t]{2}{*}{ Você se sentiu feliz em sua casa? } & Feminino & 3 & 7,89 & 6 & 15,79 & 29 & 76,31 \\
\hline & Masculino & 2 & 4,17 & 2 & 4,17 & 44 & 91,66 \\
\hline \multirow{2}{*}{ Seus pais tiveram tempo suficiente para você? } & Feminino & 5 & 13,16 & 13 & 34,21 & 20 & 52,63 \\
\hline & Masculino & 4 & 8,33 & 9 & 18,75 & 35 & 72,92 \\
\hline \multirow{2}{*}{ Seus pais tratam você de forma justa? } & Feminino & 5 & 13,16 & 19 & 23,68 & 24 & 63,16 \\
\hline & Masculino & 4 & 8,33 & 6 & 12,50 & 38 & 79,17 \\
\hline \multirow{2}{*}{$\begin{array}{l}\text { Você conversou com seus pais como você } \\
\text { gostaria? }\end{array}$} & Feminino & 9 & 23,69 & 9 & 23,68 & 20 & 52,63 \\
\hline & Masculino & 3 & 6,25 & 6 & 12,50 & 39 & 81,25 \\
\hline \multirow{2}{*}{$\begin{array}{l}\text { Você teve tempo suficiente para ficar com seus } \\
\text { amigos? }\end{array}$} & Feminino & 7 & 18,42 & 10 & 26,32 & 21 & 54,26 \\
\hline & Masculino & 3 & 6,25 & 6 & 12,50 & 39 & 81,25 \\
\hline \multirow{2}{*}{ Você realizou atividade com outros jovens? } & Feminino & 5 & 13,15 & 11 & 28,95 & 22 & 57,89 \\
\hline & Masculino & 2 & 4,17 & 13 & 27,08 & 33 & 68,75 \\
\hline \multirow{2}{*}{ Você se divertiu com seus amigos(as)? } & Feminino & 2 & 5,26 & 8 & 21,05 & 28 & 71,69 \\
\hline & Masculino & 0 & 0,00 & 5 & 10,42 & 43 & 89,58 \\
\hline \multirow{2}{*}{$\begin{array}{l}\text { Você e seus amigos(as) se ajudaram uns/umas } \\
\text { aos outros? }\end{array}$} & Feminino & 3 & 7,89 & 6 & 15,79 & 29 & 76,32 \\
\hline & Masculino & 1 & 2,08 & 7 & 14,58 & 40 & 83,34 \\
\hline \multirow{2}{*}{ Você falou o que queria com seus amigos? } & Feminino & 4 & 10,52 & 10 & 26,32 & 24 & 63,16 \\
\hline & Masculino & 1 & 2,08 & 9 & 18,75 & 38 & 79,17 \\
\hline \multirow{2}{*}{$\begin{array}{l}\text { Você sentiu que pode confiar em seus } \\
\text { amigos(as)? }\end{array}$} & Feminino & 7 & 18,42 & 12 & 31,58 & 19 & 50,00 \\
\hline & Masculino & 4 & 8,34 & 14 & 29,17 & 30 & 62,50 \\
\hline
\end{tabular}

\section{DISCUSSÃO}

Os resultados obtidos no presente estudo possibilitam a discussão de alguns pontos importantes em relação à QV na adolescência. Um primeiro aspecto se refere a uma maior participação do sexo masculino em contraposição ao feminino. Isso pode estar relacionado ao local onde o estudo ocorreu (programa social de esporte educacional), que tem uma maior participação masculina. Quando se direciona a análise para o escore geral de QV, observa-se que os adolescentes apresentaram uma percepção positiva desta, com escore superior a $66 \%$. Estudos realizados com o mesmo instrumento encontraram resultados semelhantes ${ }^{(8,10,18)}$, conforme explicitado a seguir. 
Pesquisa realizada com o instrumento Kidscreen-52 para avaliar a QVRS de 3.195 crianças e adolescentes e 2.256 pais encontrou média do escore geral de $69,64 \% \%^{(10)}$. Outro estudo, que avaliou a QV de 63 escolares do ensino fundamental de Campo Bom/RS, obteve, no escore geral, pontos médios de $50 \%{ }^{(8)}$. Em pesquisa direcionada à QVRS de latino-americanos, nos escores gerais, observaram-se médias de 62\% na Argentina, 70,06\% no Brasil e 66,91\% no Chile ${ }^{(18)}$.

Por outro lado, a percepção geral da QV obteve escores menores em estudos realizados no Chile $(45,2 \%)^{(19)}$ e na Colômbia $(45 \%)^{(20)}$.

No presente estudo, embora não se tenha desenvolvido uma metodologia de pesquisa que pudesse explicitar uma relação de causalidade direta, é importante considerar a possibilidade de um programa de esporte educacional contribuir para uma melhor percepção de QV entre os adolescentes. O programa social que serviu de campo de pesquisa é destinado a democratizar o acesso à prática e à cultura do esporte educacional. Tem como objetivo promover o desenvolvimento integral de crianças, adolescentes e jovens como fator de formação da cidadania e melhoria da $\mathrm{QV}$, prioritariamente daqueles que se encontram em áreas de vulnerabilidade social e regularmente matriculados na rede pública de ensino. Assim como a perspectiva de tentar minimizar as desigualdades e qualquer tipo de discriminação por condições físicas, sociais, de raça, cor ou de qualquer natureza que limitem o acesso à prática esportiva ${ }^{(15,16)}$.

Tendo em vista a fase de desenvolvimento biopsicossocial, para ter uma boa percepção da QV, é necessário ter um crescimento adaptativo, desenvolver-se dentro de uma dinâmica familiar coesa, que lide bem com conflitos, estar vinculado a uma rede de apoio social e afetiva, sendo importante encontrar um espaço que propicie o desenvolvimento de características individuais, como autoestima e autonomia ${ }^{(21)}$

A análise de cada domínio permitiu a identificação dos aspectos com maior e menor impacto na QV dos adolescentes, conforme explicitado anteriormente nos resultados da atual pesquisa. Nesse sentido, identificou-se que a dimensão "Sentimentos" e "Provocação/Bullying" apresentaram melhor percepção; já os domínios "Aspectos financeiros" e "Autopercepção" apresentaram as médias mais baixas.

Os resultados encontrados no presente estudo possuem semelhanças e diferenças com outras pesquisas que utilizaram o mesmo instrumento. No estudo ${ }^{(22)}$ quantitativo e qualitativo do tipo etnográfico, realizado para analisar a percepção da QVRS e a regulação da motivação de jovens jogadores de futebol de elite entre 13 a 18 anos, as melhores percepções das dimensões do Kidscreen-52 também estavam associadas às dimensões "Provocação/Bullying" e
"Sentimentos". No entanto, quanto às piores percepções, os autores $^{(22)}$ apontam "Aspectos financeiros" e "Tempo livre", diferenciando dos achados deste estudo apenas na última dimensão ${ }^{(22)}$.

$\mathrm{Na}$ pesquisa ${ }^{(21)}$ realizada com 189 adolescentes dos 14 aos 18 anos, estudantes do $2^{\circ}$ e $3^{\circ}$ ciclos e secundário do ensino regular, residentes na região do Algarve, em Portugal, verificou-se também a existência de percepções positivas nas dimensões "Provocação/Bullying", seguida dos "Amigos e apoio social", sugerindo, assim, sentimentos mais positivos face à aceitação, não provocação e respeito pelo grupo, sentimento de pertencimento, qualidade da relação e suporte percebido dos amigos. A dimensão "Ambiente escolar e aprendizagem" apresentou o resultado mais baixo ${ }^{(21)}$.

O estudo brasileiro ${ }^{(8)}$ que avaliou a QVRS de 63 adolescentes de Campo Bom/RS, com idade de 14 anos e de ambos os sexos, observou algumas diferenças nos achados. Os adolescentes da amostra apresentaram melhor percepção da QVRS nos domínios "Sentimentos" e "Amigos e apoio social", e as médias mais baixas entre os adolescentes foram nas dimensões "Provocação/Bullying" e "Estado emocional" ${ }^{\prime(8)}$.

A comparação entre os estudos ${ }^{(8,10,18-22)}$ que utilizaram o mesmo instrumento para avaliar a QV dos adolescentes coloca em hipótese que, independentemente do país ou da região em que se encontram, eles possuem percepções semelhantes, o que possibilita pensar em medidas gerais de promoção de saúde.

Especificamente em relação aos dados do presente estudo, a boa percepção dos adolescentes em relação aos "Sentimentos" e "Provocação/Bullying" pode estar relacionada ao fato de passarem boa parte do tempo no ambiente escolar e, no contraturno escolar, participarem de um programa social. Esse programa, através de múltiplas vivências esportivas, desenvolve competências sociais e proporciona o desenvolvimento integral como fator de formação da cidadania e melhoria da QV dos adolescentes participantes, favorecendo um bem-estar físico, mental e $\operatorname{social}^{(15)}$

A escola, o ambiente em que o adolescente está inserido, o suporte emocional dos pais e a referência de um adulto desempenham um papel importante no desenvolvimento social e comportamental dos adolescentes, pois esses espaços constituem-se como espaços de convivência e aprendizagem, oportunizando a socialização, proporcionando a experiência de relações de hierarquia, vivência de igualdades e convívio com as diferenças, influenciando na estrutura de formação do indivíduo ${ }^{(23)}$.

Por outro lado, a análise dos escores mais baixos encontrados nesta pesquisa, nas dimensões "Aspectos 
financeiros" e "Autopercepção", pode estar relacionada diretamente à situação de vulnerabilidade social que caracteriza o contexto de vida dos participantes, tendo em vista que estar nessa situação é um dos pré-requisitos para participação no programa social de esporte educacional no qual se coletaram os dados.

Conforme apontado anteriormente, a vulnerabilidade é um conceito multidimensional ${ }^{(24)} \mathrm{e}$, no caso dos adolescentes, está associada a aspectos sociais negativos, como: falta de garantia dos direitos e oportunidades nas áreas de educação, saúde e proteção social; envolvimento com drogas e situações de violência (doméstica e comunitária); situação de rua; trabalho infantil; condições financeiras; dificuldade geográficas, dentre outras ${ }^{(24,25)}$.

Os recursos financeiros restringem o estilo de vida; dessa forma, os adolescentes sentem-se em desvantagem financeira, e isso implica diretamente na autopercepção. Nessa dimensão, a imagem corporal é explorada por questões acerca da satisfação da aparência com roupas e outros acessórios pessoais; além disso, reflete o valor que a pessoa atribui a si própria e a percepção de quão positivamente os outros a avaliam. Uma pontuação baixa implica uma imagem corporal negativa, autorrejeição, infelicidade/insatisfação consigo próprio, baixa autoestima, desconforto com a sua aparência ${ }^{(26)}$

A análise comparativa dos dados considerando gênero apontou uma melhor percepção da QVRS para os adolescentes do sexo masculino. Esse resultado é consistente com a maioria dos estudos ${ }^{(8,11,18-22,24,26,27)}$.

Essa diferença relaciona-se com as representações simbólicas de masculinidade e feminilidade que se constroem historicamente, constatada em estudos ${ }^{(11,24)}$ que visaram compreender a QV em adolescentes. Geralmente, esse grupo etário possui a percepção de que são destinadas à mulher as tarefas domésticas, como cuidar da casa e dos irmãos menores, e aos homens reserva-se o papel das descobertas fora do ambiente doméstico e as interações sociais $^{(11,24)}$

Supõe-se que as adolescentes do sexo feminino podem estar em uma posição menos favorável em comparação aos do sexo masculino quando relacionam indicadores selecionados de saúde subjetiva e QV. Por exemplo, a aparição da puberdade e o esperado desequilíbrio hormonal reduzem as oportunidades de elas enfrentarem satisfatoriamente os eventos estressores que ocorrem nesse período da vida. Além disso, elas tendem a ser mais queixosas e questionadoras sobre sua saúde e mais exigentes em relação à sua percepção de QV. Sendo assim, ambos podem possuir as mesmas condições sociais, mas maneiras diferentes para analisar e medir distintos fatores de sua vida ${ }^{(8,18)}$.
Quanto aos domínios da QVRS, na presente pesquisa, os adolescentes do sexo masculino apresentaram melhor percepção nos domínios "Saúde e atividade física", "Sentimentos", "Estado emocional", "Autonomia e tempo livre", "Família e ambiente familiar" e "Amigos e apoio social". Em outros $\operatorname{estudos}^{(8,27)}$, eles novamente apresentaram valores superiores às adolescentes do sexo feminino nas mesmas dimensões, com exceção dos domínios "Autopercepção", "Aspectos financeiros" e "Ambiente escolar", que não apareceram.

Na dimensão "Saúde e atividade física", a diferença na percepção da QVRS entre os sexos pode ser justificada por fatores socioculturais, pois os adolescentes do sexo masculino, desde muito jovens, são estimulados a participar de atividades laborais e físicas, enquanto as adolescentes do sexo feminino são direcionadas para as atividades do $\operatorname{lar}^{(8)}$. Elas apresentam, em relação a eles, menores níveis de atividade física habitual, principalmente no que diz respeito à forma física e à disposição/energia, devido ao seu crescimento mais acelerado ${ }^{(28)}$. Elas preferem ocupar seu tempo livre de forma mais sedentária (ouvindo música, permanecendo em casa ou na casa de amigas), enquanto eles se envolvem mais em atividades desportivas, de intensidade vigorosa, e utilizam mais os recursos físicos da escola, da rua e dos espaços desportivos ${ }^{(29)}$.

A prática da atividade física é bastante importante para ambos os sexos, pois possui efeitos e benefícios para a saúde, podendo reduzir os níveis de ansiedade, estresse e depressão, aumentar o humor, o bem-estar físico e psicológico, a autoestima, o rendimento nos estudos e nas demais atividades da vida diária, influenciando positivamente na $\mathrm{QV}^{(28)}$. Além disso, é uma das formas mais eficientes para aproximar as pessoas. Atualmente, cada vez mais, é através desse momento que os adolescentes se encontram para conversar, conhecer e estreitar laços, ou mesmo aumentar seu círculo de amizades. Nesse sentido, a atividade física torna-se também uma fonte importante de satisfação no relacionamento social ${ }^{(11,29)}$.

No que diz respeito aos resultados do presente estudo sobre a percepção da dimensão "Família e ambiente familiar", os jovens do sexo masculino apresentaram percepções mais elevadas do que as jovens do sexo feminino, corroborando com outro estudo ${ }^{(24)}$. Novamente, essa diferença pode remeter à representação histórica e sociocultural construída acerca dos papéis do homem e da mulher ${ }^{(8)}$.

Segundo essa tradição, os homens devem ser fortes, independentes, agressivos, competentes e dominantes, e as mulheres devem ser dependentes, sensíveis, afetuosas, controladas, suprindo seus impulsos agressivos e sendo proibidas pela família de fazer o que querem. Sendo assim, 
o fato de o sexo feminino apresentar menor percepção nessa dimensão pode estar relacionado tanto ao maior controle parental quanto à forma de as mulheres se posicionarem mais criticamente frente às suas necessidades afetivas no seio da família ${ }^{(24)}$.

Assim, o diálogo no âmbito familiar possui importância central para que os adolescentes, independentemente do sexo, tenham a melhor adaptação possível às transformações dessa fase. Portanto, é de suma importância para esse grupo compartilhar problemas e estabelecer confiança no diálogo com os pais/responsáveis. As práticas parentais, como o interesse nas atividades dos filhos, conhecer seus amigos e o que fazem durante o tempo livre, são importantes na redução de vulnerabilidades ${ }^{(24,30)}$.

Na pesquisa ora apresentada, na dimensão "Amigos e apoio social", também houve diferença significativa entre os sexos, porém, esses resultados não são semelhantes com estudos que avaliaram a relação de amizade e apoio social entre gêneros, os quais não apontaram diferença significativa, pois ambos os sexos sentem aceitação, apoio, respeito e confiança por seus grupos de amigos de igual $\operatorname{modo}^{(20,31)}$.

Os achados do presente estudo podem estar relacionados com o fato de o sexo masculino ser mais espontâneo, vincular-se a vários grupos simultaneamente, interagir e formar amizades mais rápido do que o sexo oposto. Nesse caso, as redes de amizades do sexo masculino tendem a ser maior do que a do sexo feminino, porém, isso não implica na veracidade da amizade ${ }^{(28)}$.

As relações de amizade são o segundo patamar (o primeiro são as relações familiares) que mais influencia os níveis de percepção de bem-estar, principalmente na adolescência. Nessa fase, as amizades têm um alto grau de importância, pois o grupo exerce extrema influência no relacionamento do adolescente com o mundo, caracterizando seu jeito de ver e pensar sobre ele, sendo através dos amigos que os jovens conversam, trocam informações, tiram dúvidas e encontram liberdade para falar de assuntos que não possuem espaço no ambiente familiar ${ }^{(28,30)}$.

\section{CONCLUSÃO}

O presente estudo, realizado com adolescentes participantes de um programa social de esporte educacional, apontou uma percepção positiva de qualidade de vida de acordo com os parâmetros do instrumento utilizado. Além disso, a análise detalhada de cada dimensão avaliada explicitou que aspectos relacionados a sentimentos e provação/bullying são percebidos de forma mais positiva. Em contraposição, domínios relacionados a aspectos financeiros e autopercepção foram pontuados com médias menores.
O escore geral de QV quanto à maioria dos domínios (saúde e atividade física, sentimento, estado emocional, autonomia e tempo livre, família e ambiente familiar e amigos e apoio social) que compõem o Kidscreen-52 identificou que os adolescentes do sexo masculino apresentam melhor percepção de QV quando comparados às adolescentes do sexo feminino.

\section{REFERÊNCIAS}

1. United Nations Children's Fund, United Nations Plaza. Situação Mundial da Infância 2011: adolescência uma fase de oportunidades. New York: UNICEF; 2011.

2. Bueno CO, Strelhow MR, Camara SGW. Inserção em grupos formais e qualidade de vida entre adolescentes. Psico USF. 2010;15(3):311-20.

3. Moreira RM, Boery EM, Oliveira DC, Sales ZN, Boery RNSO, Teixeira JRB et al. Representações Sociais de adolescentes sobre qualidade de vida: um estudo de base estrutural. Ciênc Saúde Coletiva. 2015;20(1): 49-56.

4. Ministério da Saúde (BR). Estatuto da criança e do adolescente. Brasília: Ministério da Saúde; 2012 [acesso em 2015 Abr 12]. Disponível em: http:// www.crianca.mppr.mp.br/arquivos/File/publi/camara/ estatuto_crianca_adolescente_9ed.pdf.

5. Sillva MAI, Mello FCM, Mello DF. Vulnerabilidade na saúde do adolescente: questões contemporâneas. Ciênc Saúde Coletiva. 2014;19(2):627-39.

6. Pessalacia JDR, Menezes ES, Massuia DA. Vulnerabilidade do adolescente numa perspectiva das políticas de saúde pública. Bioethikos. 2010;4(4): 423-30.

7. Reis DC, Almeida TAC, Coelho AB, Madeira AMF, Paulo IMA, Alves RH. Estratégia saúde da família: atenção à saúde e vulnerabilidades na adolescência. Espaç Saúde (Online). 2014;15(1):47-56.

8. Mendes D, Piccoli JCJ, Quevedo DM. Qualidade de vida relacionada à saúde de escolares do ensino fundamental de Campo Bom, RS. Rev Bras Ciênc Movi. 2014;22(4):47-54.

9. Costa MCR. Qualidade de vida em adolescentes: um estudo no terceiro ciclo do ensino básico [tese]. Salamanca: Universidade de Salamanca; 2012.

10. Gaspar T, Matos MG. Qualidade de vida de crianças e adolescentes: versão portuguesa dos instrumentos Kidscreen-52. Cruz Quebrada: Aventura Social; 2008. 
11. Benincasa M, Custódio EM. Avaliação da qualidade de vida em adolescentes do município de São Paulo. Bol Psicol. 2011;61(134):31-42.

12. Silva MLM, Rangel LRF, Zanatta FB, Backes DS, Constenaro RGS, Piovesan C, Bertoldo JV. Indicadores de risco associados à qualidade de vida de escolares de uma comunidade vulnerável do sul do Brasil. Disciplinarum Scientia. 2013;14(1):163-71.

13. Ribeiro AMB. Qualidade de vida e risco social: estudo comparativo com alunos do $2^{\circ}$ e $3^{\circ}$ ciclo do ensino básico [tese]. Portugal: Universidade Fernando Pessoa; 2012.

14. Cabral ESM. Percepção da qualidade de vida entre jovens em risco social. Rev Enferm UFPE Online. 2013;7(8):511-9.

15. Ministério do Esporte (BR), Secretaria Nacional de Esporte, Educação, Lazer e Inclusão Social. Diretrizes 2014: Programa Segundo Tempo. Brasília: Ministério do Esporte; 2014 [acesso em 2015 Mar 10]. Disponível em: http://www.esporte.gov.br/arquivos/snelis/segun doTempo/diretrizesPSTPadrao2014.pdf.

16. Ministério do Esporte (BR), Secretaria Nacional de Esporte, Educação, Lazer e Inclusão Social. Segundo Tempo na Escola. Brasília: Ministério do Esporte; 2013 [acesso em 2015 Fev 08]. Disponível em: http:// www.esporte.gov.br/arquivos/snelis/segundoTempo/ maisEducacao/MANUAL_DE_ORIENTAES_ESPORTE_NA_ESCOLA_2013.pdf.

17. Guedes DP, Guedes JERP. Tradução, adaptação transcultural e propriedades psicométricas do KIDSCREEN-52 para a população brasileira. Rev Paul Pediatr. 2011;29(3):364-71.

18. Guedes DP, Astudillo HAV, Morales JMM, Vecino JC, Junior RP. Calidad de vida relacionada con la salud de adolescentes latinoamericanos. Rev Panam Salud Publica. 2014;35(1):46-52.

19. Molina T, Montano R, Gonzalez E. Propriedades psicométricas del cuestionario de calidad de vida relacionada con la salud Kidscreen-27 em adolescentes chilenos. Rev Méd Chile. 2014;142(11):1415-21.

20. Quiceno JM, Vinaccia S. Calidad de vida, fortalezas personales, depresión y estrés en adolescentes según sexo y estrato. Int $\mathrm{J}$ Psychol Psychol Ther. 2014;14(2):155-70.

21. Monteiro MJM. Competências para a vida em adolescentes: Avaliação da qualidade de vida relacionada com a saúde e competência social [tese]. Portugal: Universidade do Algarve; 2011.
22. Macagnan LDG. Qualidade de vida, motivação e o desempenho desportivo em jovens jogadores de futebol de elite [tese]. Portugal: Universidade do Porto; 2013.

23. Simões S, Ferreira JJ, Braga S, Vicente HT. Bullying, vinculação e estilos educativos parentais em adolescentes do $3^{\circ}$ ciclo do ensino básico. Rev Port Invest Comport Soc. 2015;1(1):30-41.

24. Rocha CAS. Percepção de suporte familiar e qualidade de vida: um estudo com adolescentes e seus pais [tese]. São Paulo: Universidade Metodista de São Paulo; 2012.

25. Morais NA, Raffaelli M, Koller SH. Adolescentes em situação de vulnerabilidade social e o continuum riscoproteção. Ava Psicol Latinoam. 2012;30(1):118-36.

26. Maximiano ASS. Acontecimentos de vida estressantes, psicopatologia e qualidade de vida em adolescentes escolarizados [tese]. Portugal: Universidade do Algarve; 2013.

27. Lino TALR. Sexualidade na adolescência: o impacto da culpa, da vergonha e do ambiente familiar, no desenvolvimento da agressividade nas manifestações sexuais nos adolescentes dos 12 aos 18 anos [tese]. Lisboa: Faculdade de Medicina da Universidade de Lisboa; 2012.

28. Santos JMC. Percepção da qualidade de vida em adolescentes (10-12 anos) de ambos os gêneros: influência da aptidão física e da atividade física [tese]. Vila Real: Universidade de Trás-os-montes e Alto Douro; 2013.

29. Lourosa APM. Qualidade de vida em adolescentes de 10-12 anos: relação com a aptidão física e os níveis de atividade física [tese]. Vila Real: Universidade de Trásos-montes e Alto Douro; 2011.

30. Reis DC, Almeida TAC, Miranda MM, Alves RH. Vulnerabilidades à saúde na adolescência: condições socioeconômicas, redes sociais, drogas e violência. Rev Latinoam Enferm. 2013;21(2):586-94.

31. Souza LK, Duarte MG. Amizade e bem-estar subjetivo. Psic Teor e Pesqui. 2013;29(4):429-36.

\section{Endereço para correspondência:}

Mirely Eunice Sobral

Universidade Federal de Pernambuco

Departamento de Terapia Ocupacional

Av. Prof. Moraes Rego, 1235

Bairro: Cidade Universitária

CEP: 50670-901 - Recife - PE - Brasil

E-mail: sobralmirely@gmail.com 